\title{
Factores individuales que protegen o favorecen el riesgo de suicidio adolescente: estudio cualitativo con grupos focales
}

\section{Individual factors that protect or favor the risk of adolescent suicide: qualitative study with focus groups}

Héctor Rubén Bravo-Andrade ${ }^{1}$ (D) Norma Alicia Ruvalcaba-Romero ${ }^{2}$ (D) , Mercedes Gabriela Orozco-Solís $^{3}$ (D), Fabiola MacíasEspinoza $^{4}$ (i)

1.Universidad de Guadalajara. Jalisco, México. Correo: ruben.bravo.a@academicos.udg.mx - https://orcid.org/0000-0002-0498-8410

2. Universidad de Guadalajara. Jalisco, México. Correo: nruval@hotmail.com - https://orcid.org/0000-0001-9209-8751

3. Universidad de Guadalajara. Jalisco, México. Correo: mercedes.orozco@cucs.udg.mx - https://orcid.org/0000-0002-0648-8233

4. Universidad de Guadalajara. Jalisco, México. Correo: fabiola.macias@cucs.udg.mx - https://orcid.org/000-0002-0865-5058

Tipología: Artículo de investigación científica y tecnológica

Para citar este artículo: Bravo-Andrade HR, Ruvalcaba-Romero NA, Orozco-Solís MG, Macías-Espinoza F. Factores individuales de riesgo y protección ante el suicidio adolescente: Estudio cualitativo con grupos focales. Duazary. 2020 enero; 17(1): 36 - 48. Doi: http://dx.doi.org/10.21676/2389783X.3220

Recibido en febrero 02 de 2019

Aceptado en junio 29 de 2019

Publicado en línea en noviembre 19 de 2019

Palabras clave: suicidio; adolecente; factores de riesgo; factores protectores; análisis cualitativo

Keywords: suicide; adolescent; risk factors; protective factors; qualitative analysis.

\section{RESUMEN}

El objetivo del presente estudio fue conocer los factores individuales asociados al riesgo y protección ante el suicidio en adolescentes de una preparatoria de la Universidad de Guadalajara, México, desde la visión de estudiantes, profesores y terapeutas. Mediante un análisis cualitativo de la información obtenida a través de los grupos focales, se encontró que los principales factores de riesgo son la depresión, el manejo inadecuado de emociones y la carencia de un sentido de vida, mientras que los factores que protegen del riesgo suicida son la capacidad de resolución de problemas, el optimismo y la autoestima. Los hallazgos se discuten desde el soporte de la literatura especializada, y así mismo se realizan recomendaciones y se proponen implicaciones prácticas.

\section{ABSTRACT}

The aim of this study was to know the individual factors associated to risk and protection on suicide in adolescents from a high school from Universidad de Guadalajara, Mexico; from a students, teachers and psychotherapist view. Through a qualitative analysis of the information got in focus groups, have found that the main risk factors are depression, inadequate emotional management and a lack of sense of life; while the protective factors from suicide are problem solving capacity, optimism and self-esteem. The findings are discussed from the support of specialized literature, and recommendations and practical implications are made. 
Factores individuales que protegen o favorecen el riesgo de suicidio adolescente: estudio cualitativo con grupos focales

\section{INTRODUCCIÓN}

El fenómeno suicida se reconoce como una problemática multifactorial y por tanto irreductible a una sola causa. En esta confluencia interactúan factores de riesgo y protección en distintos niveles, tales como el social, el familiar y el individual ${ }^{1,2}$. En aras de una mejor comprensión del fenómeno, en el presente estudio se hace énfasis en la dimensión individual, a sabiendas de que es una distinción arbitraria que permite clarificar una porción de la problemática.

Existe unanimidad en la literatura especializada en que el principal factor individual de riesgo de suicidio en población adolescente es la depresión ${ }^{3-5}$, junto con el malestar subjetivo que la acompaña, donde se incluyen: soledad, sentimientos de fracaso, vergüenza, culpa, apatía y pesimismo ${ }^{6,7}$.

Sobre esta misma línea, se ha encontrado que las dificultades que puedan presentarse en el dominio emocional contribuyen a incrementar el riesgo suicida en adolescentes. Las principales de estas son tristeza $^{8}$, sentimientos imposibles de manejar ${ }^{9}$, emociones negativas hacia sí y los demás ${ }^{10} y$, en general, la dificultad en el manejo de emociones ${ }^{11}$. Siguiendo esta perspectiva desde la subjetividad, los sentimientos de desencanto ${ }^{12}$, la desesperanza ${ }^{6,13}$ y la carencia de un sentido de vida ${ }^{14}$ se imponen como un factor considerable de riesgo para el suicidio en población adolescente.

Por otra parte, desde los factores individuales de protección ante el riesgo suicida en adolescentes, la capacidad de resolución de problemas ha mostrado ser indispensable ${ }^{8}$, especialmente cuando se trata de estrategias positivas de afrontamiento $^{1}$ y aquellas dirigidas a los problemas más que las de tipo pasivo o las encaminadas a la emoción en detrimento de la razón ${ }^{15}$.

Sumado a lo anterior, otro factor que abona a la protección ante el suicidio en población adolescente es la presencia de expectativas más o menos estables y generalizadas de que en la vida suceden cosas positivas, es decir, el optimismo, tanto para la ideación en específico ${ }^{16}$ como para la conducta suicida en general ${ }^{1,17}$.

Así también, la autoestima juega un papel fundamental cuando de contrarrestar el riesgo de suicidio se trata ${ }^{1,3,18}$. Este constructo ha sido identificado como un recurso que promueve la resiliencia ante el suicidio en jóvenes $y$ adolescentes $^{16,19}$.

Dada la multiplicidad de factores involucrados en el fenómeno suicida y la importancia de integrar aproximaciones cualitativas a su estudio con el fin de lograr una mayor comprensión de la problemática desde sus contextos específicos ${ }^{20}$, el presente estudio tuvo como objetivo conocer la visión de estudiantes, profesores y terapeutas sobre los factores individuales que protegen o ponen en riesgo de suicidio a los adolescentes de una preparatoria de la Universidad de Guadalajara, México.

\section{MATERIALES Y MÉTODOS}

\section{Tipo de investigación}

Estudio de corte cualitativo de tipo fenomenológico, cuyo propósito es comprender tanto la vivencia como la percepción del mundo del sujeto desde su realidad interna y sus marcos de referencia. En este sentido, aun cuando los participantes no hayan necesariamente experimentado en sí mismos el riesgo de suicidio, sí están en posibilidad de dar cuenta de la manera en que perciben el fenómeno suicida $^{21}$. De tal modo, se pretende explorar el contenido particular de las creencias y respuestas individuales desde el contexto y la biografía de los sujetos $^{22}$.

\section{Participantes}

El escenario de estudio fue una escuela preparatoria perteneciente a una universidad pública del estado de Jalisco, México, que se encuentra en la zona oriente de la ciudad. La localidad se puede describir como semiurbana, con un nivel socioeconómico predominantemente bajo y medio-bajo. Se realizó un muestreo por conveniencia de 22 personas agrupadas en tres grupos focales (Tabla 1). El primer grupo estuvo conformado por ocho alumnos de la preparatoria, y en él se consideraron todos los niveles, tanto del turno matutino como del vespertino (tres mujeres y cinco hombres cuyas edades oscilaron entre los 15 y los 17 años); el segundo grupo se constituyó de ocho profesores del mismo plantel escolar, sin distinción del grado o 
Héctor Rubén Bravo-Andrade, Norma Alicia Ruvalcaba-Romero, Mercedes Gabriela Orozco-Solís, Fabiola Macías-Espinoza

asignatura impartida en el plan de estudios (cuatro mujeres y cuatro hombres de entre 32 y 51 años), y en el tercer grupo participaron seis terapeutas que habían tenido contacto con el fenómeno del riesgo de suicidio en su ejercicio profesional, independientemente del enfoque clínico de trabajo (tres mujeres y tres hombres, de entre 29 y 45 años de edad).

Tabla 1. Características de los participantes por grupo focal y sexo.

\begin{tabular}{|l|l|l|l|l|l|}
\hline \multicolumn{2}{|c|}{ Alumnos } & \multicolumn{2}{c|}{ Prupos focales } \\
\hline ID & Sexo & ID & Sexo & ID & Sexo \\
\hline GFA1 & Mujer & GFP1 & Mujer & GFT1 & Hombre \\
\hline GFA2 & Hombre & GFP2 & Hombre & GFT2 & Mujer \\
\hline GFA3 & Hombre & GFP3 & Hombre & GFT3 & Mujer \\
\hline GFA4 & Mujer & GFP4 & Mujer & GFT4 & Mujer \\
\hline GFA5 & Hombre & GFP5 & Mujer & GFT5 & Hombre \\
\hline GFA6 & Hombre & GFP6 & Mujer & GFT6 & Hombre \\
\hline GFA7 & Mujer & GFP7 & Hombre & & \\
\hline GFA8 & Hombre & GFP8 & Hombre & & \\
\hline
\end{tabular}

Nota: GFA: grupo focal alumnos, GFP: grupo focal profesores, GFT: grupo focal terapeutas.

\section{Procedimiento}

La información se obtuvo entre los meses de abril y junio de 2015 con la técnica de grupos focales, dedicando una sesión para cada uno de los grupos, con una duración de entre 80 y 140 minutos. Los grupos de estudiantes y profesores se reunieron en las instalaciones de la escuela preparatoria, mientras que el de los terapeutas se llevó a cabo en un espacio externo. Todas las sesiones fueron coordinadas por el investigador principal, quien utilizó una guía de entrevista realizada ex profeso para ellas (Tabla 2). Así mismo, auxiliares en la investigación llevaron a cabo notas durante las entrevistas.

Tabla 2. Guía de preguntas de entrevista.

\section{Preguntas}

¿Qué crees que le haría pensar a un adolescente en quitarse la vida?

¿Cuáles cuestiones individuales consideras que son importantes para que una persona pueda presentar riesgo suicida?

¿Cuáles son las peores cosas que le podrían pasar a un adolescente en este momento?

¿Cómo enfrentaría un adolescente ese tipo de situaciones?

¿De qué manera crees que lidian los adolescentes con su propio riesgo suicida?

Si un adolescente estuviera en medio de un problema muy grave, ¿qué cosas lo detendrían antes de suicidarse?

¿Cuáles son las cosas que motivan a los adolescentes a vivir?

\section{Análisis de datos}

Los datos fueron transcritos a procesador de texto y posteriormente analizados con el programa MAXQDA v. 12, con el que se realizó un proceso de codificación atendiendo los pasos del método fenomenológico. Así, en primer lugar, se realizó una lectura general de la transcripción de cada grupo focal; después se delimitaron las unidades temáticas significativas, y posteriormente se determinaron categorías, en lenguaje científico, que agruparan las unidades temáticas, eliminando las redundancias. Después de analizar las transcripciones por separado se buscó la integración de todos los temas 
Factores individuales que protegen o favorecen el riesgo de suicidio adolescente: estudio cualitativo con grupos focales

centrales particulares en una estructura general ${ }^{21,23}$. Así mismo, se hizo uso de la triangulación ${ }^{24}$ entre las notas de observación y los resultados del análisis cualitativo de los grupos focales.

\section{Declaración sobre aspectos éticos}

El presente estudio se apegó a la Declaración de Helsinki de 1975 y a la Resolución 8430 de 1993 del Ministerio de Salud de Colombia para la investigación con seres humanos. Previo a las entrevistas, el investigador principal solicitó firma de consentimiento informado a los padres de los estudiantes, así como el asentimiento informado de todos los participantes para la autorización del uso de datos y la audiograbación de la sesión. La investigación fue avalada por el comité de ética de la escuela preparatoria con el número de oficio CDPTN/282/15. Este trabajo se apegó, en la medida de lo posible, a los criterios para reportar investigación cualitativa COREQ (Tabla 3).

Tabla 3. Lista de cotejo COREQ25 (Consolidated Criteria for Reporting Qualitative Research: criterios consolidados para reportar investigación cualitativa).

\begin{tabular}{|c|c|c|}
\hline N..$^{\circ}$ reactivo & Pregunta guía/descripción & Respuesta \\
\hline \multicolumn{3}{|c|}{ Dominio 1: equipo de investigación y reflexividad } \\
\hline \multicolumn{3}{|c|}{$\begin{array}{l}\text { Características personales } \\
\end{array}$} \\
\hline 1. Entrevistador/facilitador & ¿Qué autor condujo la entrevista o grupo focal? & Autor principal \\
\hline 2. Cualificación (Credentials) & ¿Cuáles eran las cualificaciones del investigador? & $\begin{array}{l}\text { Licenciado en psicología, } \\
\text { maestro en terapia } \\
\text { familiar }\end{array}$ \\
\hline 3. Ocupación & ¿Cuál era su ocupación en el momento del estudio? & Estudiante de doctorado \\
\hline 4. Género & ¿Era el investigador hombre o mujer? & Hombre \\
\hline $\begin{array}{l}\text { 5. Experiencia y } \\
\text { entrenamiento }\end{array}$ & $\begin{array}{l}\text { ¿Qué experiencia o entrenamiento tuvo el } \\
\text { investigador? }\end{array}$ & Formación profesional \\
\hline \multicolumn{3}{|l|}{ Relación con los participantes } \\
\hline $\begin{array}{l}\text { 6. Establecimiento de la } \\
\text { relación }\end{array}$ & $\begin{array}{l}\text { ¿Se estableció una relación antes del inicio del } \\
\text { estudio? }\end{array}$ & Ningún tipo de relación \\
\hline $\begin{array}{l}\text { 7. Conocimiento de los } \\
\text { participantes del investigador }\end{array}$ & $\begin{array}{l}\text { ¿Qué sabían los participantes sobre el entrevistador? } \\
\text { Ej. Metas personales, razones para hacer la } \\
\text { investigación. }\end{array}$ & $\begin{array}{l}\text { Los participantes fueron } \\
\text { informados del objetivo } \\
\text { de la investigación bajo } \\
\text { lineamientos éticos }\end{array}$ \\
\hline $\begin{array}{l}\text { 8. Características del } \\
\text { entrevistador }\end{array}$ & $\begin{array}{l}\text { ¿Qué características se informaron sobre el } \\
\text { entrevistador/facilitador? }\end{array}$ & $\begin{array}{l}\text { Se presentó como } \\
\text { investigador }\end{array}$ \\
\hline \multicolumn{3}{|l|}{ Dominio 2: diseño del estudio } \\
\hline \multicolumn{3}{|l|}{ Marco teórico } \\
\hline $\begin{array}{l}\text { 9. Orientación teórica y } \\
\text { metodológica }\end{array}$ & $\begin{array}{l}\text { ¿Qué orientación metodológica se estableció para } \\
\text { sustentar el estudio? }\end{array}$ & Introducción y método \\
\hline \multicolumn{3}{|l|}{ Selección de los participantes } \\
\hline 10. Muestreo & ¿Cómo fueron seleccionados los participantes? & Método (participantes) \\
\hline 11. Abordaje metodológico & ¿Cómo fue el acercamiento a los participantes? & Cara a cara \\
\hline 12. Tamaño de muestra & ¿Cuántos participantes hubo en el estudio? & Método (participantes) \\
\hline 13. No participación & $\begin{array}{l}\text { ¿Cuántas personas se negaron a participar o } \\
\text { abandonaron? ¿Razones? }\end{array}$ & 0 \\
\hline \multicolumn{3}{|l|}{ Escenario } \\
\hline $\begin{array}{l}\text { 14. Escenario de la } \\
\text { recolección de datos }\end{array}$ & ¿Dónde se recolectó la información? & Método (Procedimiento) \\
\hline $\begin{array}{l}\text { 15. Presencia de no } \\
\text { participantes }\end{array}$ & $\begin{array}{l}\text { ¿Había alguien más presente, además de los } \\
\text { participantes y el investigador? }\end{array}$ & $\begin{array}{l}\text { Auxiliares de } \\
\text { investigación }\end{array}$ \\
\hline 16. Descripción de la muestra & $\begin{array}{l}\text { ¿Cuáles son las características relevantes de la } \\
\text { muestra? }\end{array}$ & $\begin{array}{l}\text { Método (participantes, } \\
\text { tabla 1). }\end{array}$ \\
\hline
\end{tabular}


Héctor Rubén Bravo-Andrade, Norma Alicia Ruvalcaba-Romero, Mercedes Gabriela Orozco-Solís, Fabiola Macías-Espinoza

\begin{tabular}{|c|c|c|}
\hline \multicolumn{3}{|l|}{ Recolección de datos } \\
\hline 17. Guía de entrevista & $\begin{array}{l}\text { ¿Hubo guía de preguntas provista por los autores? } \\
\text { ¿Hubo prueba piloto? }\end{array}$ & $\begin{array}{l}\text { Método (procedimiento, } \\
\text { tabla 2), no hubo piloto }\end{array}$ \\
\hline 18. Entrevistas repetidas & $\begin{array}{l}\text { ¿Se llevaron a cabo entrevistas repetidas? Si es así, } \\
\text { ¿cuántas? }\end{array}$ & No \\
\hline 19. Grabación de audio/video & ¿Se grabó audio o video para la recolección de datos? & $\begin{array}{l}\text { Sí, método } \\
\text { (procedimiento) }\end{array}$ \\
\hline 20. Notas de campo & $\begin{array}{l}\text { ¿Se hicieron notas de campo durante o después de la } \\
\text { entrevista o grupo focal? }\end{array}$ & Sí \\
\hline 21. Duración & $\begin{array}{l}\text { ¿Cuál fue la duración de las entrevistas o grupos } \\
\text { focales? }\end{array}$ & Método (procedimiento) \\
\hline 22. Saturación de datos & ¿Se discutió la saturación de datos? & No \\
\hline $\begin{array}{l}\text { 23. Devolución de } \\
\text { transcripciones }\end{array}$ & $\begin{array}{l}\text { ¿Las transcripciones fueron devueltas a los } \\
\text { participantes para comentarios y/o corrección? }\end{array}$ & No \\
\hline \multicolumn{3}{|l|}{ Dominio 3: análisis y hallazgos } \\
\hline \multicolumn{3}{|l|}{ Análisis de datos } \\
\hline $\begin{array}{l}\text { 24. Número de codificadores } \\
\text { de datos }\end{array}$ & ¿Cuántos codificadores analizaron los datos? & Autor principal \\
\hline $\begin{array}{l}\text { 25. Descripción del árbol de } \\
\text { codificación }\end{array}$ & $\begin{array}{l}\text { ¿Proveen los investigadores una descripción del árbol } \\
\text { de codificación? }\end{array}$ & Sí \\
\hline 26. Derivación de temas & $\begin{array}{l}\text { ¿Los temas fueron identificados previamente } o \\
\text { derivaron de los datos? }\end{array}$ & $\begin{array}{l}\text { Ambos. Método (análisis } \\
\text { de datos) }\end{array}$ \\
\hline 27. Software & $\begin{array}{l}\text { ¿Qué software, si corresponde, se utilizó para analizar } \\
\text { los datos? }\end{array}$ & $\begin{array}{l}\text { MAXQDA 12. Método } \\
\text { (análisis de datos) }\end{array}$ \\
\hline $\begin{array}{l}\text { 28. Comprobación del } \\
\text { participante }\end{array}$ & $\begin{array}{l}\text { ¿Los participantes proporcionaron comentarios sobre } \\
\text { los hallazgos? }\end{array}$ & No \\
\hline \multicolumn{3}{|l|}{ Informe } \\
\hline 29. Citas presentadas & $\begin{array}{l}\text { ¿Se presentaron citas de los participantes para ilustrar } \\
\text { los temas/hallazgos? ¿Se identificó cada cita? }\end{array}$ & $\begin{array}{l}\text { Resultados. Sí, por } \\
\text { categoría para cada } \\
\text { grupo focal }\end{array}$ \\
\hline $\begin{array}{l}\text { 30. Consistencia entre datos y } \\
\text { hallazgos }\end{array}$ & $\begin{array}{l}\text { ¿Hubo consistencia entre los datos presentados y los } \\
\text { hallazgos? }\end{array}$ & $\begin{array}{l}\text { Sí. Se presentan en la } \\
\text { discusión }\end{array}$ \\
\hline $\begin{array}{l}\text { 31. Claridad de los temas } \\
\text { principales }\end{array}$ & $\begin{array}{l}\text { ¿Los temas principales se presentaron claramente en } \\
\text { los hallazgos? }\end{array}$ & Resultados y discusión \\
\hline $\begin{array}{l}\text { 32. Claridad de los temas } \\
\text { menores }\end{array}$ & $\begin{array}{l}\text { ¿Hay una descripción de casos diversos o discusión de } \\
\text { temas menores? }\end{array}$ & Resultados y discusión \\
\hline
\end{tabular}

\section{RESULTADOS}

De las notas de campo obtenidas durante la realización de los grupos focales, los temas que más destacaron fueron la falta de habilidades de resolución de problemas y la carencia de proyecto de vida como favorecedores de riesgo suicida, mientras que los principales elementos que protegen al adolescente de dicho riego son las habilidades de resolución de problemas y la autoestima.

El análisis de la información obtenida en los grupos de discusión giró en torno a los elementos que desde el ámbito individual se perciben como favorecedores o protectores del suicidio en adolescentes. A continuación, se detalla cada uno con sus respectivas categorías (Figura 1). 


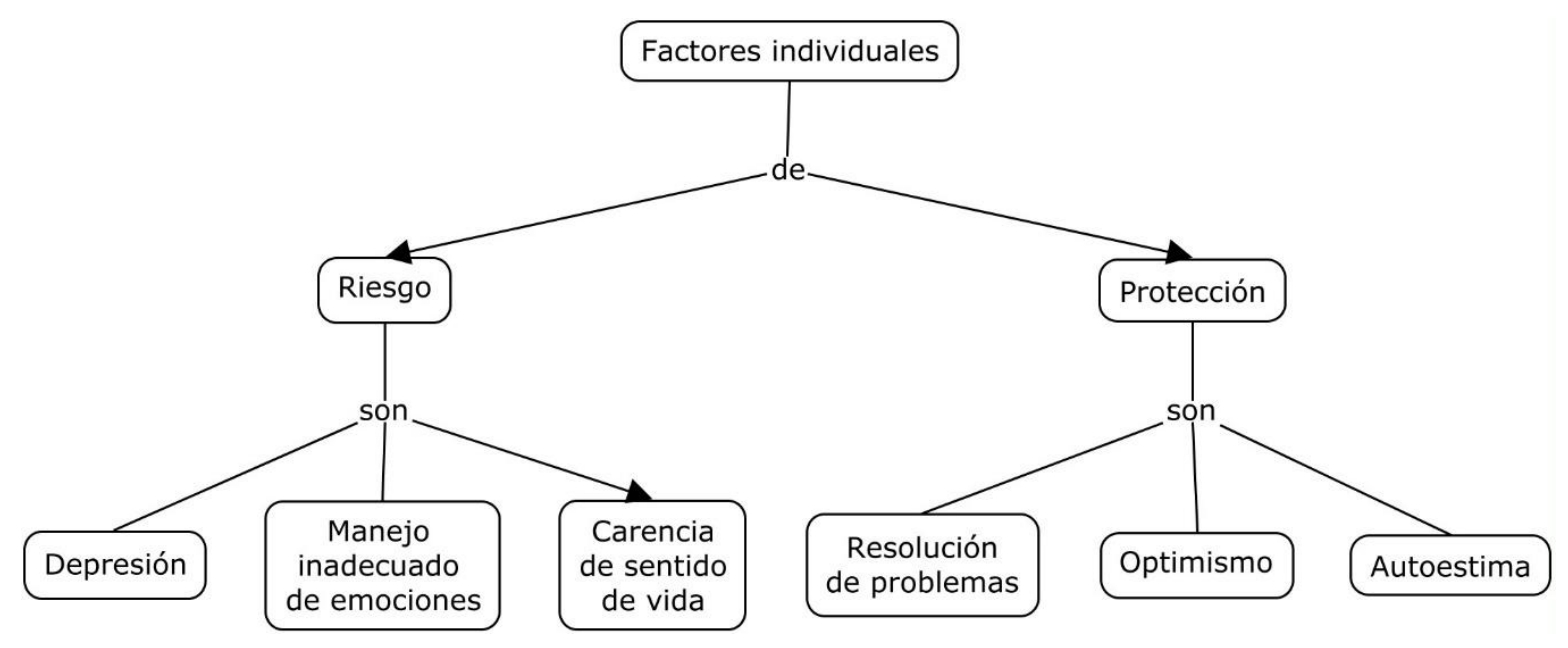

Figura 1. Árbol de códigos de factores individuales asociados al suicidio en adolescentes.

Factores de riesgo. En este tema se distinguieron las categorías: depresión, manejo inadecuado de emociones y carencia de sentido de vida.

Depresión. En los tres grupos focales se identificó este padecimiento como el principal responsable del riesgo suicida. En torno a esta categoría surgieron ideas relacionadas con el aislamiento, el enojo y la sensación de soledad.

En el grupo de alumnos se puso de manifiesto que la depresión se encuentra ligada a la falta de expresión de emociones:

Un joven podrá sentirse con deseos suicidas porque, al sentirse solo, incomprendido, porque no habla, la persona se cierra, sufre en silencio, nunca se desahoga. Pienso que las probabilidades de suicidio son más posibles, son más grandes que alguien que sí habla con sus seres queridos, un amigo o hasta consigo mismo (GFA8).

El grupo de profesores hizo énfasis en la sensación de vacío como principal característica del malestar depresivo:

Creo que lo que los lleva a eso es que sienten que no tienen afecto, o no les llena nada: ni la escuela, ni la familia, ni nada. Entonces ese vacío me imagino que es lo que los orilla a eso. Aunque a simple vista parezcan normales y actúen como... pues como el resto... pero precisamente esa necesidad de estar saciando algo que no tienen es lo que les impulsa (GFP5).

Por su parte, en el grupo de terapeutas se presentó la idea de que en la depresión confluyen una serie de emociones desbordadas:

En este caso, la tristeza, podemos decir, frustración, hasta el enojo, también. Pero que al estar disparadas ya no hay inteligencia emocional, ya no están controladas (GFT6).

Manejo inadecuado de emociones. En esta categoría apareció en los tres grupos focales la idea de fluctuaciones en el estado de ánimo de los adolescentes, así como respuestas violentas contra otros y contra sí mismos.

Para el grupo de alumnos la adolescencia misma se identifica con cierta inestabilidad:

Es la etapa más frágil porque somos como muy moldeables, como que estamos muy sensibles para ver hacia qué lado nos vamos (GFA4).

En el grupo de profesores se mencionó que los cambios pueden ser indicadores de riesgo de suicidio en los estudiantes:

Cambio de humor, que de repente está bien el muchacho pero que de repente saca arranques de ira o algo (GFP7). 
Los terapeutas, por su parte, destacaron la presencia del enojo como un indicador de riesgo. Incluso se hizo referencia a que el suicidio puede utilizarse como una especie de castigo o venganza:

Pareciera ser que cuando se habla de suicidio, se habla de tristeza, de desesperanza, y todo eso, pero parece ser que también hay otro elemento - se dijo hace rato-: es el enojo. "Estoy enojado contigo. Para que tú sufras, me mato" (GFT6).

Carencia de sentido de vida. Los principales elementos encontrados en esta categoría se relacionan con la incertidumbre existencial, la desesperanza y la búsqueda de inmediatez en la satisfacción.

En los alumnos se observó una marcada tendencia a la búsqueda de identidad, acompañada de incertidumbre:

Más bien eso es como el problema de no encontrarse a sí mismo y tener como esa crisis de existencia del no saber quién eres y de que no te corresponde un lugar (GFA4).

Los profesores hicieron mención de que el sentido de una vida futura se encuentra marcado por la incertidumbre y la desesperanza:

Yo veo que hay mucha perdida de esperanza en el futuro. Muchos están en prepa pero a lo mejor salen de la prepa y se van a trabajar. Yo pienso que en el momento en que ellos tengan una esperanza real de salir de la escuela y a lo mejor continuar con otros estudios, o tener acceso a un buen trabajo, un trabajo "chido" como dicen, yo creo que eso haría retomar el rumbo de ellos y su confianza. Yo pienso que una persona que no tiene esperanza, pues ahí se generan muchos problemas desde los económicos, pérdida de valores personales (GFP3).

En el grupo de terapeutas surgió la noción de que el sentido de vida se encuentra ligado a la posesión material y la inmediatez:

Esto de que le pregunte uno a un joven "¿Qué quieres ser de grande?" y diga "sicario" obedece a que a la oportunidad de trabajo y de decir "Pues ¿sabes qué? Más vale vivir bien, ser feliz, aunque sea un poco y tener una camioneta así, que un proyecto que sea estudiar o a lo mejor tener un trabajo remunerado de acuerdo al estudio que se tenga" (GFT2).

Factores de protección. En este rubro se distinguieron la resolución de problemas, el optimismo y la autoestima.

Resolución de problemas. En esta categoría emergió que una de las formas de resolución de conflictos es la capacidad de buscar ayuda. Así también se acentuó la importancia de contar con distintas formas de afrontamiento de las dificultades.

Entre los alumnos se destacó el reconocimiento de que existe un problema como primer paso para su resolución:

Creo que lo primero es aceptar que hay un problema, porque muchas veces quien tiene problemas no lo acepta, y ese ya es un problema, y al aceptarlo buscarle las soluciones posibles (GFA8).

Los profesores privilegiaron el diálogo como medio para la resolución de problemas:

Yo creo que eso de la comunicación sí es muy importante y que es una alternativa que solucionaría muchos problemas (GFP5).

Entre los terapeutas se mencionó que estas habilidades pueden ser empíricamente contrastables y al mismo tiempo son aprendidas:

Sería como habilidades muy medibles, como la capacidad de desarrollar estrategias de solución de problemas exitosas, que tiene que ver con múltiples modelos interiorizados en su familia, o en su entorno inmediato, y que esas habilidades de resolución de problemas llevan a buscar otras opciones (GFT2).

Optimismo. En esta categoría destacó que con una actitud positiva se puede aprender de los errores y abonar a la resolución de los problemas.

En la discusión con alumnos emergió la importancia de centrarse en las cuestiones positivas de las vivencias: 
Factores individuales que protegen o favorecen el riesgo de suicidio adolescente: estudio cualitativo con grupos focales

Yo creo que sería darle más importancia a las cosas buenas que a las cosas malas. Como quedarte o seguir viviendo quizás por una persona, o porque puedes estar soñando o estar pensando en lo que vas a hacer ya más grande o que vas a ser otra vez, o sea, que te vas a sentir mejor, pues, de lo que te estas sintiendo ahora, de que puedes o a la mejor están incitando a que no vas a llegar a nada y tú dices: "No, yo sé que sí puedo". Tú mismo echarte como la mano, ¿no?, en pensar que sí puedes, que puedes llegar a hacer lo que te propongas (GFA1).

En el grupo de profesores se antepuso una visión positiva generalizada:

Yo creo que actuar positivo ante todo (GFP7).

Mientras que entre los terapeutas se hizo mención de que el optimismo puede incidir en creer que las cosas pueden ser diferentes y por ende reducir el riesgo de suicidio:

Sí, tal vez, "esto puede cambiar", entonces, porque ahí puede el riesgo disminuir un poco (GFT5).

Autoestima. Entre las ideas que se vincularon con esta categoría se encuentran sentirse capaces de realizar actividades de manera adecuada, aceptarse y quererse a sí mismos.

Para los alumnos, el principal elemento destacado dentro de la autoestima fue el amor a sí mismo:

A lo mejor encontrar el amor, pero en tu interior y no buscarlo en las demás personas, porque, bueno, como dicen, no puedes encontrarlo en otras personas si no 10 encuentras primero en ti mismo (GFA7).

En el grupo de los profesores se hizo referencia al reconocimiento de las propias capacidades:

Un autorreconocimiento, decir "Soy bueno para esto y por lo tanto busco tal cuestión" (GFP5).

Por su parte, los terapeutas destacaron que el concepto de sí mismo que pueda tener el adolescente tiene un componente relacional:
Creo que sí tiene que ver mucho con el autoconcepto, cómo se sienten ellos. Pero no solamente ellos; creo que siempre tiene en relación al otro, y ese otro es y cómo se hace sentir a través de otro (GFT1).

\section{DISCUSIÓN}

El presente estudio tuvo como objetivo conocer las visiones de estudiantes, profesores y terapeutas sobre los factores individuales que se perciben como favorecedores o protectores del suicidio en adolescentes de preparatoria de una universidad pública de Guadalajara, Jalisco, México. Conforme se profundiza en las categorías, y sobre todo en aquello que las conecta, parece revelarse un hilo conductor que tiene que ver con el juego entre las nociones de incertidumbre y la seguridad, que se manifiesta en distintos niveles.

En primer lugar, destaca que aparentemente el riesgo de suicidio en adolescentes, en sintonía con esta etapa, circunda alrededor de una inestabilidad que va más allá de no tener certeza sobre el futuro, sino que se extiende al grado de experimentar fragilidad en las propias emociones. La principal forma en que esto se manifiesta es a través de emociones violentas. Al respecto, Lucio-Gómez y Hernández-Cervantes ${ }^{4}$ han encontrado una asociación entre la hostilidad y el riesgo suicida en adolescentes. En esta misma línea, Hernández y Lucio $^{29}$ aseguran que en las mujeres se relaciona el enojo y resentimiento con el riesgo de suicidio, mientras que en los hombres se vincula a un pobre control del enojo. Por su parte, De la Roca ${ }^{30}$ ha reportado el papel de la culpa y el enojo en los suicidios adolescentes, en tanto que Orri et al. ${ }^{10}$ encontraron que en algunos casos el intento de suicidio se lleva a cabo como una forma de venganza, con el fin de resolver un malestar interno surgido por un conflicto relacional.

En segundo término, la incertidumbre se manifiesta en el posicionamiento ante la vida. El mundo se percibe desde una visión pesimista y sin sentido, lo que puede incrementar la presencia de ideación suicida, tal como se ha reportado en la literatura ${ }^{6,7,12,31,32,33}$. La desesperanza que despierta tal carencia empuja a tratar de dotar de sentido a la vida a través de la inmediatez. Esta, sin embargo, es 
una fútil empresa que fracasa al no encontrar una base de solidez que sirva como contención, ya que cuando un adolescente prioriza en su proyecto de vida un desarrollo individual, puede desplegar signos de desilusión hacia el entorno, manifestando posturas materialistas y hedonistas, padeciendo un estado de confusión sobre su vida ${ }^{34}$. Ante esto, resulta interesante la apreciación de Jiménez et al. ${ }^{13}$ en cuanto a que las conductas de riesgo suponen un medio para enfrentar los sentimientos de desesperanza, narcisismo y aislamiento social. Es decir, en un intento por encontrar sentido a la vida, se la pone en riesgo.

Vale la pena destacar que la depresión fue atribuida en los grupos tanto a la desarmonía emocional como al vacío existencial antes descritos, así como al malestar subjetivo ${ }^{6,7,26,27}$, lo que pone de manifiesto la íntima relación entre todos estos elementos. No está por demás mencionar el amplio sustento que se encuentra en la literatura entre la depresión y el suicidio $^{3-5}$.

Ahora bien, la incertidumbre no solo proviene del mundo o del futuro, sino desde sí mismo ${ }^{33}$. Después de todo, ¿cómo puede encontrarse sentido a la vida si no se tiene primero sentido de sí mismo? La ideación suicida guarda una estrecha relación con la manera en que el adolescente se percibe. En este juego de inestabilidades, la discrepancia entre una imagen idealizada de sí mismo y la imagen que de hecho tiene el adolescente de sí configura una oposición intolerable que puede aproximar hacia el suicidio, tal como se describe en varones jóvenes que han consumado el suicidio ${ }^{35}$.

Por otra parte, la seguridad se genera en torno a la autoestima, la cual se soporta en una identidad clara y estable. El sentido de identidad $-\mathrm{y}$ su adecuada síntesis - se ve favorecido por las relaciones significativas de los adolescentes, asociándose de manera negativa con conductas autolesivas ${ }^{36}$. Existe consenso en la literatura en que la autoestima es, en general, un factor protector en la adolescencia ${ }^{37-39}$, algo que es especialmente cierto para el caso de riesgo de suicidio. Esta noción ha encontrado amplio sustento empírico ${ }^{16,18,19,40,41}$. En este sentido, Jiménez et al. ${ }^{13}$ afirman que la autoestima, además de fungir como un factor protector ante la ideación suicida, se asocia con vidas más activas, mejor control sobre las circunstancias, menor ansiedad y mayor capacidad para tolerar el estrés interno y externo.

A su vez, la autoestima permite la seguridad que soporta la resolución de problemas a través del manejo adecuado de emociones. Al respecto, Vírseda et $a .^{15}$ aseguran que las estrategias de afrontamiento centradas en el problema y la búsqueda de apoyo, contrarias a aquellas de tipo pasivo, o centradas en la emoción, son más favorables en detrimento de la ideación suicida. De hecho, Rivera-Heredia et $a .^{42}$ sostienen que la capacidad de pedir ayuda es un recurso protector de sintomatología depresiva. Así también, SánchezTeruel y Robles-Bello ${ }^{19}$ plantean que las habilidades para pedir apoyo y ayuda inmediata son un factor protector que promueve la resiliencia ante el suicidio en adolescentes. De esta forma, la adecuada capacidad de resolución de problemas protege al adolescente del riesgo de suicidio ${ }^{8}$.

En este sentido, un adolescente que tiene amor por sí mismo, en consonancia, gozará de la sensación de autocontrol en el nivel afectivo ${ }^{19,31,32}$ y generará emociones positivas protectoras ${ }^{17}$. La inteligencia emocional, de manera específica, la capacidad de comprensión de las emociones, la tolerancia al estrés y el control de impulsos actúan como factores protectores contra el suicidio ${ }^{32}$.

Es digno de destacar que el optimismo es otro de los factores centrales que protegen del riesgo suicida a los adolescentes ${ }^{1,33}$. La psicología positiva, de manera progresiva, ha ido acrecentando sus aportaciones para la comprensión de las conductas suicidas $^{17}$, encontrando soporte en distintos autores como Quiceno y Vinaccia ${ }^{43}$ y Sánchez-Teruel et al. ${ }^{16}$. Aunque vale la pena destacar que es necesario enriquecer la literatura con estudios que aborden esta relación.

Por último, una adecuada autoestima proporciona una sensación de seguridad hacia el mundo y permite elaborar un sentido la vida. De tal suerte, se hace necesaria la vivencia de ciertas experiencias que doten de significado la vida de las personas ${ }^{37}$. Específicamente en el caso de los adolescentes, un proyecto de vida se forja a partir de la búsqueda de autonomía $^{36}$. De este modo, contar con un sentido de vida enriquece las capacidades resilientes de los adolescentes ante el suicidio ${ }^{14,19}$. 
En síntesis, una apreciación positiva de sí mismo, aunada al reconocimiento y gestión de las propias emociones, favorece la resolución de problemas de manera satisfactoria para sí y los demás, permitiendo generar experiencias que doten de sentido la propia vida, proporcionando seguridad y disminuyendo la incertidumbre y la posibilidad de sufrir malestar depresivo. Tal conclusión armoniza con el planeamiento de que la salud mental positiva confiere resiliencia contra la ideación y la conducta suicida ${ }^{44}$.

Entre las fortalezas de la presente investigación, destaca la profundidad del tratamiento de los datos, así como presentar la gama de factores en su complejidad. Por otro lado, se reconoce como limitante el tamaño de la muestra, así como la falta de triangulación con los participantes. Se recomienda llevar a cabo investigaciones en las que se contrasten empíricamente las relaciones entre los factores descritos en este estudio. Así mismo, estos hallazgos pueden servir como sustento para la elaboración de programas de prevención del riesgo suicida en adolescentes que tomen en consideración las categorías aquí detalladas.

\section{AGRADECIMIENTOS}

Al Consejo Nacional de Ciencia y Tecnología de México por la beca de manutención N. ${ }^{\circ} 375718$ para estudios de doctorado del primer autor, de donde surge el presente estudio.

\section{DECLARACIÓN SOBRE CONFLICTOS DE INTERESES}

Los autores declaran que no existe conflicto de intereses.

\section{CONTRIBUCIÓN DE LOS AUTORES}

Primer autor: diseño de proyecto, trabajo de campo, análisis de datos, elaboración del manuscrito y redacción.

Segundo autor: diseño metodológico, elaboración del manuscrito y redacción.

Tercer autor: revisión de codificaciones, elaboración del manuscrito y redacción.

Cuarto autor: elaboración del manuscrito y redacción.

\section{REFERENCIAS BILIOGRÁFICAS}

1. Organización Mundial de la Salud (OMS). Prevención del suicidio: un imperativo global [internet]. Washington DC: OMS; 2014. Disponible en: http://apps.who.int/iris/bitstream/handle/106 65/136083/9789275318508_spa.pdf;jsessionid =CA81B89EAA7A1C301B52301A3E6DCABB?seq uence $=1$.

2. González-Forteza C, Arana D, Jiménez J. Problemática suicida en adolescentes y el contexto escolar: Vinculación autogestiva con los servicios de salud mental. Salud Ment [revista en la internet]. 2008; 31(1): 23-27. Disponible

en: http://revistasaludmental.mx/index.php/salud _mental/article/view/1206/1204.

3. González-Forteza C, Ramos L, Caballero MA, Wagner F. Correlatos psicosociales de depresión, ideación e intento suicida en adolescentes mexicanos. Psicothema [revista en la internet]. 2003; 15(4): 524-532. Disponible en: http://www.psicothema.es/pdf/1102.pdf.

4. Lucio-Gómez E, Hernández-Cervantes Q. Personalidad y riesgo suicida en adolescentes estudiantes. Rev Med Inst Mex Seguro Soc [revista en la internet]. 2009; 47(1): 33-S40. Disponible en: http://www.medigraphic.com/pdfs/imss/im2009/ims091g.pdf.

5. Pavez P, Santander N, Carranza J, Vera-Villarroel P. Factores de riesgo asociados a la conducta suicida en adolescentes con trastorno depresivo. Rev Med Chile. 2009; 137(2): 226233. http://dx.doi.org/10.4067/S003498872009000200006

6. Balcázar P. Soledad como predisponente al suicidio en la adolescencia. En: Castillo M. (Coord.) Salud mental, sociedad contemporánea. Toluca: UAEM; 2000. 131-135.

7. Casullo M. Ideaciones y comportamientos suicidas en adolescentes: una urgencia social. Anu Investig [revista en la internet]. 2004; 12: 173-182. Disponible

en: 
http://www.scielo.org.ar/pdf/anuinv/v12/v12a 17.pdf.

8. Barón O. Adolescencia y suicidio. Psicología desde el Caribe. 2000; 6: 48-69. Disponible en: https://www.redalyc.org/articulo.oa?id=21300 605.

9. Nizama M. Suicidio. Rev Perú Epidemiol [revista en la internet]. 2011; 15(2): 81-85. Disponible en:

https://www.redalyc.org/pdf/2031/203122516 002.pdf.

10. Orri $M$, Paduanello $M$, Lachal J, Falissard $B$, Sibeoni J, Revah-Levy A. Qualitative approach to attempted suicide by adolescents and young adults: The (neglected) role of revenge. Plos One. $\quad 2014 ; \quad 9(5)$ : 1-8. https://doi.org/10.1371/journal.pone.0096716.

11. Chavez-Hernández A, González-Forteza C, Juárez $A$, Vázquez $D$, Jiménez $A$. Ideación y tentativas suicidas en estudiantes del nivel medio del estado de Guanajuato, México. Acta Universitaria [revista en la internet]. 2015; 25(6): 43-50. Disponible en: http://www.scielo.org.mx/pdf/au/v25n6/20079621-au-25-06-00043.pdf.

12. Rivera $M, \quad$ Andrade P. Conductas autodestructivas e intento suicida en los adolescentes. En: Andrade P, Cañas J, Betancourt D. (Comps.). Investigaciones psicosociales en adolescentes. Chiapas: UNICACH; 2008. 89-110.

13. Jiménez A, Mondragón L, González-Forteza C. Self-esteem, depressive symptomatology, and suicidal ideation in adolescents: results of three studies. Salud Ment [revista en la internet]. 2007; 30(5): 20-26. Disponible en: http://www.revistasaludmental.mx/index.php/ salud_mental/article/view/1186/1184.

14. Villalobos-Galvis F, Arévalo C, Rojas F. Adaptación del Inventario de Resiliencia ante el Suicidio (SRI-25) en adolescentes y jóvenes de Colombia. Rev Panam Salud Públ [revista en la internet]. 2012; 31(3): 233-239. Disponible en: https://www.scielosp.org/article/rpsp/2012.v3 1n3/233-239/.
15. Vírseda J, Amado G, Bonilla M, Gurrola G. Afrontamiento e ideación suicida en adolescentes. Revista psicología.com [revista en la internet]. 2011, 15: 1-18. Disponible en: http://www.psiquiatria.com/bibliopsiquis/asset store/44/24/56/4424564394162973335486290 2892216181529.

16. Sánchez-Teruel D, García-León A, MuelaMartínez J. Relación entre alta ideación suicida y variables psicosociales en estudiantes universitarios. Electronic Journal of Research in Educational Psychology. 2013; 11(2): 429-450. http://dx.doi.org/10.14204/ejrep.30.13013

17. Galarza A. Aportes de la psicología positiva a la comprensión y el abordaje de los comportamientos suicidas. Psicodebate. 2015; 16(1): 9-26. http://dx.doi.org/10.18682/pd.v16i1.564.

18. Ceballos-Ospino G, Suárez-Colorado Y, SuescúnArregocés J, Gamarra-Vega M, González E, Sotelo-Manjarres A. Ideación suicida, depresión y autoestima en adolescentes escolares de Santa Marta. Duazary [revista en la internet]. 2015; 12(1): 15-22. Disponible en: http://revistas.unimagdalena.edu.co/index.php /duazary/article/view/1394/795.

19. Sánchez-Teruel D, Robles-Bello M. Factores protectores que promueven la resiliencia ante el suicidio en adolescentes y jóvenes. Pap Psicol [revista en la internet]. 2014; 35(2): 181-192. Disponible en: http://www.papelesdelpsicologo.es/pdf/2438. pdf.

20. Santana R, Sánchez L, Quintanilla R, Padrós F. Suicidología de corte cualitativo en Latinoamérica: Revisión de métodos reportados en una década. Uaricha [revista en la internet]. 2016; 13(30): 149-168. Disponible en: http://www.revistauaricha.umich.mx/ojs_uaric ha/index.php/urp/article/view/127/130.

21. Martínez M. Comportamiento humano. México: Trillas; 2001.

22. Smith J. Beyond the divide between cognition and discourse: Using interpretative phenomenological analysis in health psychology. Psychology \& Health. 1996; 11(2): 
Factores individuales que protegen o favorecen el riesgo de suicidio adolescente: estudio cualitativo con grupos focales

261-271.

https://doi.org/10.1080/08870449608400256.

23. Taylor S, Bogdan R. Introducción a los métodos cualitativos de investigación. Barcelona: Paidós; 1987.

24. Okuda Benavides, M, Gómez-Restrepo, C. Métodos en investigación cualitativa: triangulación. Revista Colombiana de Psiquiatría [revista en la internet]. 2005; 34(1):118-124. Disponible en: http://www.redalyc.org/articulo.oa?id=806284 03009.

25. Tong A, Sainsbury P, Craig J. Consolidated criteria for reporting qualitative research (COREQ): a 32-item checklist for interviews and focus groups: International Journal of Quality and Health Care. 2007; 9(6): 349-357. https://doi.org/10.1093/intqhc/mzm042.

26. Arancibia M, Behar R. Alexitimia y depresión: evidencia, controversias e implicancias. Rev Chil Neuro-psiquiatr. $\quad 2015$; 53(1): $24-34$. http://dx.doi.org/10.4067/S071792272015000100004.

27. Restrepo-Restrepo C, Vinaccia S, Quiceno M. Resiliencia y depresión: un estudio exploratorio desde la calidad de vida en la adolescencia. Suma Psicológica [revista en la internet]. 2011; 1(2): 41-48. Disponible en: http://www.scielo.org.co/pdf/sumps/v18n2/v1 8n2a04.pdf.

28. Hernández Q, Lucio E. Modelo de evaluación del riesgo suicida adolescente basado en la exposición a eventos estresantes y configuraciones de personalidad. En: Eguiluz L, Córdova M, Rosales J. (Comp.). Ante el suicidio. Su comprensión y tratamiento. México: Pax; 2010. 117-136.

29. De la Roca J. Psicoanálisis y epidemiología del suicidio en Guanajuato de 1997 a 2001. Reflexiones sobre jóvenes. Salud Pública Méx [revista en la internet]. 2008; 50(1): 4-5. Disponible en: http://www.scielo.org.mx/scielo.php?script=sci _arttext\&pid=S0036-36342008000100004.

30. Suárez Y, Restrepo D, Caballero C. Ideación suicida y su relación con la inteligencia emocional en universitarios colombianos. Revista Universitaria Industrial de Santander Salud. 2016; 48(4): 470-478. http://dx.doi.org/10.18273/revsal.v48n42016005.

31. Mamani-Benito O, Brousettt-Minaya M, CoriZúñiga D, Villasante-Idme K. (2018). La inteligencia emocional como factor protector en adolescentes con ideación suicida. Duazary. 2018; 15(1): 39-50. http://dx.doi.org/10.21676/2389783X.2142

32. Li, D, Zhang, R, Liu, S, Liu, J, Zhang, T. The Characteristics of Chinese Adolescents With Suicidal Ideation: A Mixed-Methods Approach. Journal of Adolescent Research. 2017; 34(2): 201-230.

https://doi.org/10.1177/0743558417723603.

33. Barrón M. Proyecto de vida en los jóvenes. En: Cardozo, G. (Coord.), Adolescencia Promoción de salud y resiliencia. Córdoba: Brujas; 2005. 213-225.

34. Rassmusen, $M$, Dyregrov, $K$, Haavind, $H$, Leenaars, A, Dieserud, $G$. The role of self-esteem in suicides among young men. OMEGA - Journal of Death and Dying. 2018; 77(3): 217-239. https://doi.org/10.1177/0030222815601514.

35. Gandhi, A, Claes, L, Bosmans, G, Baetens, I, Wilderjans, T, Maitra, S, Kiekens, G, Luyckx, K. Non-Suicidal Self-Injury and Adolescents Attachment with Peers and Mother: The Mediating Role of Identity Synthesis and Confusion. J Child Fam Stud. 2016; 25(6): 17351745. https://doi.org/10.1007/s10826-0150350-0.

36. Kotliarenco M, Cáceres I, Fontanilla M. Estado de arte en resiliencia [internet]. Washington DC: Organización Panamericana de la Salud; 1997. Disponible en: http://www1.paho.org/hq/dmdocuments/200 9/Resil6x9.pdf.

37. Organización Mundial de la Salud. El suicidio, un problema grave de salud pública enorme y sin embargo prevenible, según la OMS [internet]. 2004 Disponible en: http://www.who.int/mediacentre/news/releas es/2004/pr61/es/. 
38. Páramo M. Factores de riesgo y factores de protección en la adolescencia: Análisis de contenido a través de grupos de discusión. Ter. Psicol. 2011; 29(1): 85-95. http://dx.doi.org/10.4067/S071848082011000100009.

39. González-Forteza C, Juárez C, Montejo L, Oseguera $G$, Wagner $F$, Jiménez $A$. Ideación suicida y su asociación con drogas, depresión e impulsividad en una muestra representativa de estudiantes de secundaria del estado de Campeche, México. Acta Universitaria [revista en la internet]. 2015; 25(2): 29-34. Disponible en:

https://www.redalyc.org/articulo.oa?id=41648 310005.

40. Sánchez-Sosa J, Villareal-González M, Musitu G, Martínez B. Ideación suicida en adolescentes: Un análisis psicosocial. Interven Psicosocial [revista en la internet]. 2010; 19(3): 279-287. Disponible en: https://www.redalyc.org/articulo.oa?id=17981 7507008 .

41. Rivera-Heredia $M$, Andrade P. Recursos individuales y familiares que protegen al adolescente del intento suicida. Rev Intercont Psicol Educ [revista en la internet]. 2006; 8(2): 23-40. Disponible en: https://www.redalyc.org/pdf/802/80280203.p df.

42. Quiceno J, Vinaccia S. Calidad de vida, factores salutogénicos e ideación suicida en adolescentes. Ter Psicol. 2013; 31(2): 263-271. http://dx.doi.org/10.4067/S071848082013000200012.

43. Brailovskaia J, Teismann T, Margraf J. Cyberbullying, positive mental health and suicide ideation/behavior. Psychiatry Research. 2018; 267: 240-242. https://doi.org/10.1016/j.psychres.2018.05.07 4. 